\title{
Brachial Plexus Neurofibroma: A Case Report
}

\author{
Mu Ha Lee, Hyung Ki Park, Hye Ran Park, Sukh Que Park, Jae Chil Chang, Sung Jin Cho \\ Department of Neurosurgery, Soonchunhyang University Seoul Hospital, Soonchunhyang University College of Medicine, \\ Seoul, Republic of Korea
}

Corresponding author:

Hyung Ki Park

Department of Neurosurgery, Soonchunhyang

University Seoul Hospital, Soonchunhyang University

College of Medicine, 59 Daesagwan-ro, Yongsan-gu,

Seoul 04401, Republic of Korea

Tel: +82-2-709-9267

Fax: +82-2-792-5976

E-mail: phk007@schmc.ac.kr

Received: April 2, 2020

Revised: April 10, 2020

Accepted: April 14, 2020
The brachial plexus is an anatomically complex structure. Tumors that originate from the brachial plexus are rare and present clinical challenge for neurosurgeons due to the anatomical complexity. We present the case of 59-year-old male patient with right hand weakness who was diagnosed with a neurogenic tumor originating from brachial plexus. He underwent wide the excision of tumor through the anterior supraclavicular approach. Postoperatively, the patient developed right arm numbness and motor weakness. At 2 month following the removal of tumor, he recovered normal function.

Key Words: Brachial plexus; Nerve sheath neoplasms; Neurofibroma; Peripheral nerves

\section{INTRODUCTION}

Peripheral nerve tumors are a heterogeneous group of mostly benign tumors that are rare in the general population. These tumors can be divided into 2 groups, peripheral nerve sheath and non-neural sheath tumors. Neurofibromas are composed of a mix of Schwann cells, perineurial-like cells, and fibroblasts, interspersed with nerve fibers, wire-like strands of collagen, and a myxoid matrix ${ }^{7)}$. The majority of neurofibromas are solitary(up to 90\%) and not associated with neurofibromatosis type $1^{7)}$. Neurofibromas account for $5 \%$ of all benign soft tissue tumors. Tumors of the brachial plexus region are only infrequently encountered by most neurosurgeons. These tumors comprise less than $5 \%$ of tumors of the hand and upper extremities".

The management of brachial plexus tumors requires a complete understanding of the anatomy, and a surgeon needs to understand a patient's clinical presentation, pathologic variation, and surgical techniques for operation ${ }^{5}$. Therefore, to help improve upon treatment of the brachial plexus tumor, we report on a case of a neurofibroma from the brachial plexus.

\section{CASE REPORT}

A 59-year-old man presented with a slow growing painless mass in the right supraclavicular region. Although the mass was painless, he had been suffering from a tingling sensation and radiating pain on the right ulnar side of the forearm for three years. On physical examination, he had a firm mass on the right supraclavicular region. He had right hand weakness (hand grasping grade $4+/ 5$ ). His personal medical history was without any remarks and there was no family history of a similar tumor.

In the enhanced computed tomography scans, a $5.7 \times 4.4$ $\times 4.5 \mathrm{~cm}$-sized low density peripheral enhancing fusiform - shaped mass was observed on the right side and behind the clavicle (Fig. 1A). An enhanced T1-weighted axial neck magnetic resonance imaging (MRI) showed a well - margined mass with a fluid-fluid level and peripheral enhancing (Fig. 1B). In the enhanced T1-weighted coronal MRI, the mass that was originating from the right of the C8-T1 lower trunk compressed toward the upper and middle trunk of the brachial plexus (Fig. 1C).

The patient underwent a wide excision of the tumor through an anterior supraclavicular approach. An oblique incision parallel to the lateral border of the sternocleidomastoid (SCM) from just below the mastoid process to the clavicle with a transverse limb laterally at the level of the clavicle was made to form zigzag. The platysma was divided to and develop subplatysmal flaps. The SCM muscle was checked and divided. The omohyoid muscle was identified, and the well-capsulized tumor mass below the omohyoid muscle was identified. It compress toward the upper and middle trunk of the brachial plexus (Fig. 2). Dissection and adhesiolysis were performed carefully due to severe adhesion to the adjacent tissue. After that, because the size of the tumor was too large to be removed all at once, the tumor was punctured for internal decompression and debulking. Residual tumor was removed after further dis- 

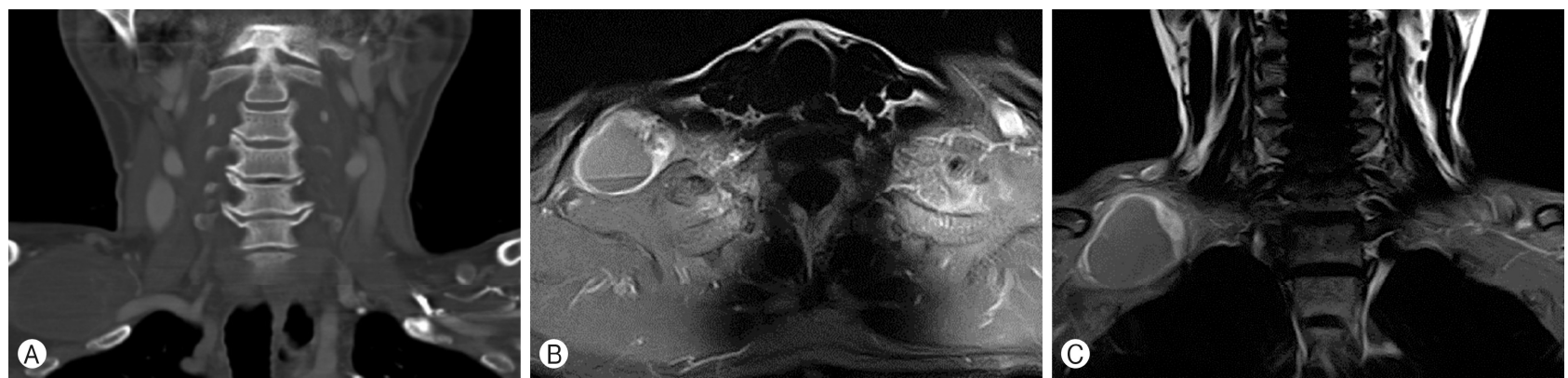

Fig. 1. A coronal neck computed tomography (enhanced) image show a $5.7 \times 4.4 \times 4.5 \mathrm{~cm}$ low attenuating fusiform-shaped mass $(A)$. In the enhanced $\mathrm{Tl}$-weighted axial magnetic resonance imaging (MRI), a high signal mass with fluid-fluid level was observed (B). An enhanced T1-weighted coronal MRI showed a peripheral enhancing mass that originated from the lower trunk and compressed toward the upper and middle trunk of the brachial plexus (C).

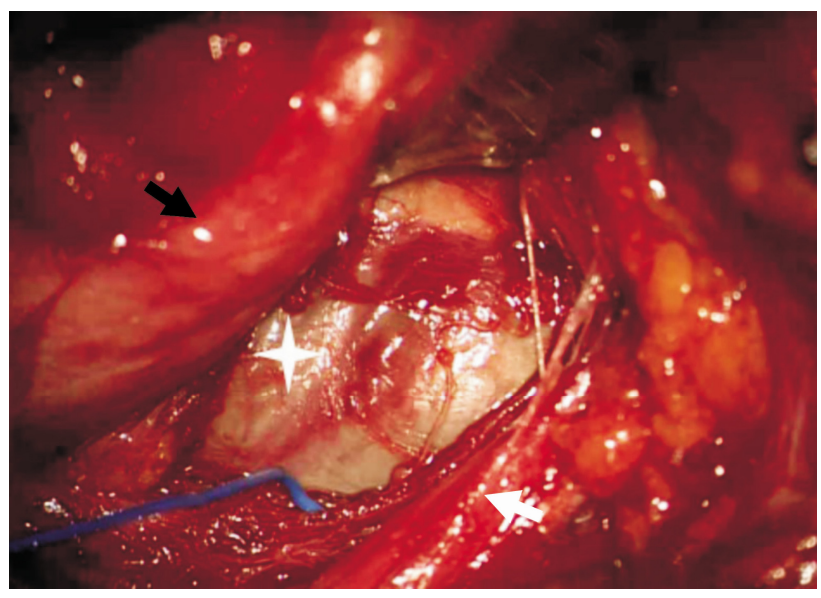

Fig. 2. Intraoperative findings during tumor mass removal. Well capsulized tumor mass (white star) was exposed and found to originate from the lower trunk of brachial plexus. The tumor compressed upwardly both the upper (white arrow) and middle (black arrow) trunk of brachial plexus.

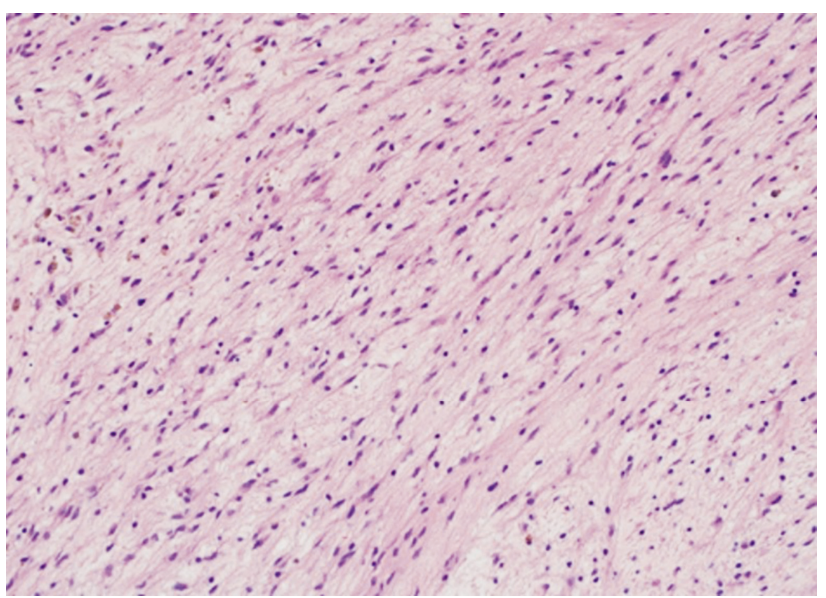

Fig. 3. Light microscopic exam showed the proliferation of spindle cells on a loose myxoid background (hematoxylin and eosin stain $[\mathrm{H \& E}], \times 200$ magnification). section. During the operation, intraoperative monitoring was performed and there was no significant signal change.

The tumor was pathologically diagnosed as a neurofibroma. Histologically, the tumor was composed of spindle shaped cells on a loose myxoid background (Fig. 3). Immunohistochemical analysis showed expression of the S-100 protein and Vimentin in tumor cells.

Postoperatively, the patient developed right arm numbness and motor weakness (hand grasping motor grade 4). After 2 months, the patient recovered normal function.

\section{DISCUSSION}

Tumors of the brachial plexus are relatively rare and present a clinical challenge for neurosurgeons ${ }^{3)}$. Tumors derived from perineural Schwann cells include schwannomas, neurofibromas, and malignant peripheral nerve sheath tumors. Secondary tumors involving peripheral nerves include locally invasive or metastatic carcinoma, lymphoma, and sarcoma ${ }^{6}$. Neurofibroma are composed of a mix of Schwann cells, perineurial-like cells, and fibroblasts, interspersed with nerve fibers, wire-like strands of collagen, and a myxoid matrix. The majority is solitary (up to $90 \%$ ) and not associated with neurofibromatosis type $1^{7)}$.

Symptoms and signs of peripheral nerve tumors are caused by direct nerve invasion, involvement of surrounding tissues, or mass effects ${ }^{6}$. The clinical presentation of brachial plexus tumors in patients is often variable but can include a painless palpable mass, local or radiating pain, motor loss, sensory loss, paresthesias, and dyspnea. According to Huang et al. ${ }^{5)}$, the most common presenting symptom is pain (70\%), followed by sensory change (61\%) and motor loss (52\%). The duration and progression of symptoms or signs are important, as most benign tumors have a longer duration and a slow rate of progression, while malignant tumors tend to progress rapidly in size, pain, and neurologic deficits ${ }^{1}$. Thus, a careful history and physical examination are necessary for the diagnosis and management of brachial plexus-region tumors.

In the case of brachial plexus lesions, an MRI with contrast 
is the study of choice to delineate the margins of the tumor from surrounding tissues ${ }^{2}$. According to Go et al. ${ }^{4)}$, brachial plexus tumors show a well-defined mass with the long axis in line with the nerve of origin and a homogeneous intermediate signal intensity on T1-weighted sequences. The tumors were hyperintense on T2 weighted images with a homogenous central low signal intensity (target sign) and strong enhancement following contrast administration ${ }^{4}$. The presence of the target sign does not allow the differentiation of a neurofibroma from a schwannoma'). However, schwannomas and neurofibromas can be distinguished by determining the relationship of the tumor to the nerve of origin. In the case of a schwannoma, the nerve has an asymmetric relationship with the tumor, whereas the nerve passes through the center of a neurofibroma or is obliterated by $\mathrm{it}^{8}$. Fine needle aspiration procedures or biopsies are often performed, but these procedures could be dangerous due to the complexity of brachial plexus, possible damage to the intact fascicle or possible hemorrhage ${ }^{5)}$.

The choice of surgical approach is important and can be selected according to the size of the tumor, its location, and its relationship with adjacent organs. An anterior supraclavicular approach has been used to treat most tumors involving the roots and trunks ${ }^{5}$. The incision is centered on the posterior triangle and is, either transverse or L-shaped. During exposure, important landmarks include the SCM muscle, the supraclavicular fat pad, the omohyoid muscle, phrenic nerve, and anterior scalene muscle ${ }^{5}$. The infraclavicular approach has been used for lesions involving the lower plexus root or trunk involved by the tumor, and patients who have had a history of radiotherapy or resection ${ }^{3}$. For tumors involving both the supraclavicular and infraclavicular portions of the brachial plexus, the combined or transclavicular approaches could been used ${ }^{5)}$.

Regardless of the approach selected, the operative steps for the resection of most neural sheath tumors are similar. The proximal and distal aspects of the tumor are identified, and the tumor is isolated from the surrounding neurovascular structures $^{3)}$. Intraoperative monitoring of nerve action potentials, compound muscle action potential and, in some cases, somatosensory evoked potential can play a useful role in the surgical management of brachial plexus region tumors" ${ }^{5}$. Combined with an awareness of the tumor's anatomic and pathologic involvement of the nerve fascicles, monitoring these potentials can obviate or minimize injury to healthy nerve fascicles and permit complete tumor extirpation ${ }^{5)}$.

Although the success rate for the surgical resection of brachial plexus tumors is high, complications can arise. Paresthesias, weakness, and postoperative pain generally recover with time and physical therapy. A common complication of the supraclavicular approach for tumor resection is phrenic nerve paralysis, which usually improves with times).

\section{CONCLUSION}

From this case, we learned that when we encounter a supraclavicular mass, neurogenic tumors should be considered in the differential diagnosis. The resection of tumors is the choice treatment for most tumors originating from the brachial plexus. The appropriate surgical approach is an intraoperative microsurgical dissection technique with understanding of the complex anatomy of the brachial plexus and intraoperative monitoring is required to improve patient outcome.

\section{CONFLICTS OF INTEREST}

No potential conflict of interest relevant to this article was reported.

\section{REFERENCES}

1. Baehring JM, Betensky RA, Batchelor TT: Malignant peripheral nerve sheath tumor: the clinical spectrum and outcome of treatment. Neurology 61:696-698, 2003

2. Binder DK, Smith JS, Barbaro NM: Primary brachial plexus tumors: imaging, surgical, and pathological findings in 25 patients. Neurosurg Focus 16:E11, 2004

3. Das S, Ganju A, Tiel RL, Kline DG: Tumors of the brachial plexus. Neurosurg Focus 22:E26, 2007

4. Go MH, Kim SH, Cho KH: Brachial plexus tumors in a consecutive series of twenty one patients. J Korean Neurosurg Soc 52:138-143, 2012

5. Huang JH, Zaghloul K, Zager EL: Surgical management of brachial plexus region tumors. Surg Neurol 61:372-378, 2004

6. Mrugala MM, Batchelor TT, Plotkin SR: Peripheral and cranial nerve sheath tumors. Curr Opin Neurol 18:604-610, 2005

7. Pilavaki M, Chourmouzi D, Kiziridou A, Skordalaki A, Zarampoukas T, Drevelengas A: Imaging of peripheral nerve sheath tumors with pathologic correlation: pictorial review. Eur J Radiol 52:229-239, 2004

8. Saifuddin A: Imaging tumours of the brachial plexus. Skeletal Radiol 32:375-387, 2003

9. Varma DG, Moulopoulos A, Sara AS, Leeds N, Kumar R, Kim EE, et al.: MR imaging of extracranial nerve sheath tumors. J Comput Assist Tomogr 16:448-453, 1992 